\title{
GAUSSIAN BROADCAST CHANNELS WITH COOPERATING RECEIVERS: THE SINGLE COMMON MESSAGE CASE
}

\author{
S. Lasaulce \\ CNRS - Supélec \\ Laboratoire des Signaux et Systèmes \\ 3 rue Joliot Curie, 91192 Gif-sur-Yvette, France
}

\author{
A.G. Klein* \\ Cornell University \\ School of Elec. \& Computer Engr. \\ Ithaca, NY 14853, USA
}

\begin{abstract}
The main purpose of this paper is to evaluate the benefits of receive cooperation in a broadcast situation where two receivers want to decode the same message. Additionally the cooperation channel is assumed to be orthogonal to the downlink channels. In the case where the cooperation channel is unidirectional, the channel capacity is determined and the performance loss induced by orthogonalizing the cooperation channel is evaluated. In the bidirectional and orthogonal case lower and upper bounds for the capacity are provided. More specifically two decoding schemes are compared. Coding scheme 1: the two receivers use estimate-and-forward. Coding scheme 2: each receiver uses, in a complementary manner, estimate-and-forward a certain fraction of the time and decode-and-forward for the rest of the time. For realistic levels of the cooperation powers the performance of the second scheme is shown to be close to the considered upper bound.
\end{abstract}

\section{INTRODUCTION}

In the conventional broadcast channel (BC), one transmitter sends messages to several receivers, each of which operates independently. In this paper we focus on a class of broadcast channels with cooperating receivers. We use the term cooperative broadcast channel (CBC) to refer to this channel. Such a situation is particularly relevant in densely populated cellular environments where subscribers' mobile stations (MS) could cooperate in order to enhance the overall network performance.

The idea of cooperating transmitters has been introduced in [1] for multiple access channels (MACs). This channel has experienced a recent resurgence of interest especially through the concept of cooperative diversity [2], [3]. From these works it is seen that cooperation leads to an increase in rate (broader rate region) and more robust systems where users' achievable rates are less sensitive to channel variations. The cited references focus on the uplink case, i.e. the MAC with cooperating transmitters. In this paper we focus on cooperation for the downlink scenario. The most recent results concerning the discrete cooperative $\mathrm{BC}$ have been given in [4] and [5]. In [4] the authors treated the general (discrete) $\mathrm{CBC}$ with a bidirectional conference link, which is a bidirectional noiseless channel with finite capacities (see also [1] for more details). The extension of this work leads to the the case where the cooperation channel is orthogonal. In [5] the authors addressed the relay broadcast channel, which corresponds to the case where the (non-orthogonal) cooperation channels are classical noisy channels and the receivers act as standard relay

\footnotetext{
* Supported in part by Applied Signal Technology and NSF Grants CCF0310023 and INT-0233127.
}

nodes. In both cases the authors made significant progress regarding the problem under consideration by providing useful inner and outer bounds for the capacity region. However the channel capacity for the special case of the single common message is not determined. This case is nevertheless of practical interest (e.g. a satellite transmitting to cooperating TV receivers, a cellular network with a common channel, a sensor network).

Therefore, based on the results of [4] and [5], we investigated the Gaussian BC with a single common message and an orthogonal cooperation channel. We will first provide the capacity expression for the $\mathrm{CBC}$ with a single common message and a unidirectional cooperation channel (section 3 ). Then by orthogonalizing the cooperation channel and optimally allocating the bandwidth (similarly to [6] for the relay channel) we will be able to evaluate the exact performance loss due to orthogonalization. In section 4 we will analyze the bidirectional case and discuss the "best" choice for the coding/decoding scheme. Before tackling these issues we first describe in section 2 the signal model for the Gaussian CBC.

\section{SIGNAL MODEL}

The transmitter encodes the common message $W \in\left\{1, \ldots, 2^{n R}\right\}$ ( $R$ is the coding rate) intended for both users 1 and 2 into the signal $X \sim \mathcal{N}(0, P)$. In practice $X$ is transmitted on a block by block basis, which can be written as $\underline{x}=\left(x_{1}, \ldots, x_{n}\right)=f(w)$, $n$ is the block/codeword length. The received signals are denoted by $Y_{1}, Y_{2}$. Additionally the cooperative signals are denoted by $X_{12} \sim$ $\mathcal{N}\left(0, P_{12}\right), X_{21} \sim \mathcal{N}\left(0, P_{21}\right)$. With these notations the received signals write as:

$$
\left\{\begin{array}{l}
Y_{1}=X+X_{21}+Z_{1} \\
Y_{2}=X+X_{12}+Z_{2}
\end{array}\right.
$$

where $Z_{1} \sim \mathcal{N}\left(0, N_{1} B\right), Z_{2} \sim \mathcal{N}\left(0, N_{2} B\right)$ are classical AWG noises and $B$ is the available bandwidth. This signal model will only be used in section 3 (i.e. $X_{21} \equiv \varnothing$ ) to be compared to the orthogonal case. For the rest of the time the signal model under consideration (see Figure 1) will assume the cooperation channel to be orthogonal to the downlink channels. The signal model becomes:

$$
\left\{\begin{array}{lll}
Y_{1}=X+Z_{1} & Y_{12}=X_{12}+Z_{12} \\
Y_{2}=X+Z_{2} & Y_{21}=X_{21}+Z_{21}
\end{array}\right.
$$

where $Z_{i j} \sim \mathcal{N}\left(0, N_{i j} B_{i j}\right), I\left(Y_{i} ; Y_{j i}\right)=0$. Without loss of generality we assume that the orthogonality condition is met thanks to frequency duplexing between the downlink and interuser channels. The total bandwidth is split into three bands of frequency. In order to describe the corresponding bandwidth allocation we intro- 
duce the triplet $\underline{\alpha}=\left(\alpha_{0}, \alpha_{12}, \alpha_{21}\right): B=B_{0}+B_{12}+B_{21}=$ $\left(\alpha_{0}+\alpha_{12}+\alpha_{21}\right) B$. At last we assume that $N_{i j}=N_{j}$.

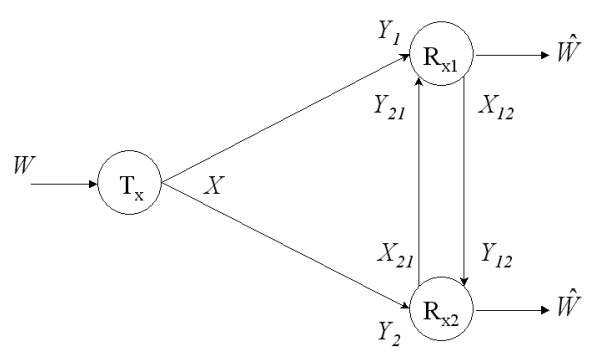

Fig. 1. CBC with an orthogonal cooperation channel

\section{GAUSSIAN CBC WITH UNIDIRECTIONAL COOPERATION CHANNEL}

In this section we assume $X_{21} \equiv \varnothing$. Although less general than its bidirectional counterpart the unidirectional case is still of practical interest. For example in a cellular context it can happen that the instantaneous receive SNRs are very different (because of fast fading) and the main part of performance improvement will be essentially due to the receiver being in the best reception conditions at the considered time (or data block).

Even though the capacity is not known both for the discrete and Gaussian relay channels (RC) the capacity of the channel under consideration can be found both in the discrete and Gaussian cases, the Gaussian case being the case of interest here. In the case under investigation receiver 1 does not only act like a simple relay node but has also to decode a message, which is a different situation from that of the relay channel. Technically speaking, the capacity of the general RC is defined from only one decoding constraint $\operatorname{Pr}\left[g_{2}\left(\underline{Y_{2}}\right) \neq\right.$ $W] \rightarrow 0$ while the $\mathrm{CBC}$ with a single common message is defined from two decoding constraints $\left(\operatorname{Pr}\left[g_{1}\left(\underline{Y_{1}}\right) \neq W\right] \rightarrow 0\right.$ and $\left.\operatorname{Pr}\left[g_{2}\left(\underline{Y_{2}}\right) \neq W\right] \rightarrow 0\right)$. This specificity provides us with an additional Fano's inequality. Exploiting this observation one can then prove, along the lines of [7], that decode-and-forward is optimum without assuming any explicit form of degradedness [7]. For the general (discrete) CBC we have the following theorem.

Theorem 3.1 The capacity of the BC having a unidirectional cooperation channel for the single common message case is given by:

$$
C=\sup _{p\left(x, x_{12}\right)} \min \left\{I\left(X ; Y_{1} \mid X_{12}\right), I\left(X, X_{12} ; Y_{2}\right)\right\} .
$$

We note that this expression is the same as for the physically degraded relay channel, though here we have made no degradedness assumption (we did not assume that $p\left(y_{1}, y_{2} \mid x, x_{12}\right)=p\left(y_{1} \mid x, x_{12}\right)$ $\left.p\left(y_{2} \mid x, y_{1}\right)\right)$. This is useful because the Gaussian signal models, (1) and (2) with $X_{21} \equiv \varnothing$, are not physically degraded. Therefore, by applying theorem 3.1 in the Gaussian case and using the power constraints $E\left(X^{2}\right) \leq P, E\left(X_{12}^{2}\right) \leq P_{12}$ we get the channel capacity instead of an achievable rate.

\subsection{Capacity for the non-orthogonal case}

In the non-orthogonal case the downlink and cooperation channels share the same band of frequency whose bandwidth is $B$. Using
Theorem 3.1 and the power constraints $E\left(X^{2}\right) \leq P, E\left(X_{12}^{2}\right) \leq$ $P_{12}$ we get:

$$
C_{\overline{\text { orth }}}=\max _{r \in[0,1]} \min \left\{C\left(\frac{r P}{N_{1} B}\right), C\left(\frac{P+P_{12}+2 \sqrt{\bar{r} P P_{12}}}{N_{2} B}\right)\right\}
$$

where $C(x)=B \log _{2}(1+x)$ and $\bar{r}=1-r$.

If $N_{2} \leq N_{1}$ the capacity is simply $C\left(\frac{P}{N_{1} B}\right)$. On the other hand, if $N_{2}>N_{1}$ there are two possible working regimes [7]. If $P_{12} \geq P \frac{N_{2}-N_{1}}{N_{1}}$ then $r^{*}=1$ and $C_{\overline{\text { orth }}}=C\left(\frac{P}{N_{1} B}\right)$. Now if $P_{12}<P \frac{N_{2}-N_{1}}{N_{1}}$ the best $r$ is given by: $\bar{r}^{*}=\frac{a_{1}^{2}+a_{3}^{2} \pm 2 \sqrt{a_{1}^{2} a_{3}^{2}}}{a_{2}^{2}}$ where $r^{*} \in[0,1], a_{0}=P+P_{12}, a_{1}=\sqrt{P P_{12}}, a_{2}=P \frac{N_{2}}{N_{1}}, a_{3}^{2}=$ $a_{1}^{2}+a_{2}^{2}-a_{0} a_{2}$. A sufficient condition for $a_{1}^{2}+a_{2}^{2}-a_{0} a_{2}$ being non-negative is precisely $N_{2} \geq N_{1}$. An important point to notice here is that the saturation regime is reached for a finite cooperation power $P_{12}^{*}=P \frac{N_{2}-N_{1}}{N_{2}}$.

\subsection{Capacity for the orthogonal case}

For a given bandwidth allocation $\left(\alpha_{0}, \alpha_{12}=1-\alpha_{0} \triangleq \bar{\alpha}_{0}\right)$ the capacity expresses as: $C=\min \left\{R_{1}\left(\alpha_{0}\right), R_{2}\left(\alpha_{0}\right)\right\}$ where $R_{1}\left(\alpha_{0}\right)=$ $\alpha_{0} C\left(\rho_{1} / \alpha_{0}\right), R_{2}\left(\alpha_{0}\right)=\alpha_{0} C\left(\rho_{2} / \alpha_{0}\right)+\bar{\alpha}_{0} C\left(\rho_{12} / \bar{\alpha}_{0}\right)$, and $\rho_{i}=$ $P /\left(N_{i} B\right), \rho_{i j}=P /\left(N_{i j} B\right)$. In this paper we consider that the parameter $\alpha_{0}$ is not fixed, then the capacity is obtained by optimizing the bandwidth allocation: $C_{\text {orth }}=\max _{\alpha_{0}} \min \left\{R_{1}\left(\alpha_{0}\right), R_{2}\left(\alpha_{0}\right)\right\}$. It can be shown that there is a unique $\alpha_{0}^{(m)} \in[0,1]$ maximizing $R_{2}\left(\alpha_{0}\right)$. Depending on the channel parameters the optimum bandwidth allocation $\alpha_{0}^{*}$ is either given by $\alpha_{0}^{(m)}$ or the intersection of $R_{1}($.$) and R_{2}($.$) . Without loss of generality assume now$ that $N_{1}<N_{2}$. A quite natural question is then to ask what is the cooperation power needed for being in the saturation regime i.e. $C_{\text {orth }}=C\left(\rho_{1}\right)$. For a given $\alpha_{0}, R_{2}\left(\alpha_{0}\right) \geq R_{1}\left(\alpha_{0}\right)$ is equivalent to: $\rho_{12} \geq \bar{\alpha}_{0}\left[\left(\frac{1+\rho_{1}}{1+\rho_{2}}\right)^{\alpha / \bar{\alpha}_{0}}-1\right]$. For a fixed $\alpha_{0} \neq 0$ the required cooperation power is clearly finite. On the other hand if we are interested in optimizing bandwidth allocation, $\alpha_{0}$ can take all the values between 0 and 1 . When $\alpha_{0} \rightarrow 1$ the saturation condition becomes $\rho_{12} \geq \exp \left\{\frac{1}{\bar{\alpha}_{0}}\left[\ln \left(\frac{1+\rho_{1}}{1+\rho_{2}}\right)+\bar{\alpha}_{0} \ln \bar{\alpha}_{0}\right]\right\}$. Since $\rho_{1}>\rho_{2}$ it is clear that the cooperation power has to be infinite for $\bar{\alpha}_{0} \rightarrow 0$. Contrary to the non-orthogonal case, one cannot reach the saturation regime for finite cooperation powers and there will always be a performance loss due to orthogonalizing the interuser channel. One of the purposes of the following subsection is precisely to assess this loss for a typical scenario.

\subsection{Numerical results}

Orthogonal case: optimum bandwidth allocation.

We chose $N_{1} B=N_{21} B=1, N_{2} B=N_{12} B=4, P=10$. The table below gives the optimum parameter $\alpha_{0}^{*}$ as a function of the cooperation power for $\frac{P_{12}}{P} \in[-20 \mathrm{~dB}, 80 \mathrm{~dB}]$. We see that $\alpha_{0}^{*} \rightarrow 1$ in two cases: 1 . when the cooperation power is very low, which is logical; 2 . when the cooperation power tends to infinity. The interpretation of this case is that the cooperation channel is very efficient and little bandwidth is needed for achieving the maximum performance.

\begin{tabular}{|c|c|c|c|c|c|c|}
\hline$\frac{P_{12}}{P}[d B]$ & -20 & -10 & -1.5 & 0 & 10 & 80 \\
\hline$\alpha_{0}^{*}$ & 0.99 & 0.91 & 0.59 & 0.62 & 0.80 & 0.95 \\
\hline
\end{tabular}


Performance loss due to orthogonalization.

We always have $N_{1} B=N_{21} B=1, N_{2} B=N_{12} B=4$. For three different values of the transmit power $P \in\{1,10,100\}$, figure 2 represents the relative capacity loss due to orthogonalization as a function of $P_{12}: \Delta C[\%] \triangleq 100\left(C_{\overline{\text { orth }}}-C_{\text {orth }}\right) / C_{\overline{\text { orth }}}$. The performance loss is clearly driven by the ratio $P_{12} / P$. If this ratio is greater than $20 \mathrm{~dB}$ the relative capacity loss is less than $10 \%$ for the considered range of transmit powers. In the real life such a situation can appear when the link budget corresponding to the cooperation channel is much better than that corresponding to the downlink channels, which is in fact a very common scenario in a cellular networks (e.g. 2 users in the same room or building). On the other hand if the available cooperation power is very limited the non-orthogonal solution performs much better than its orthogonal counterpart. Of course, in practice, complexity and feasibility issues have also to be accounted for.

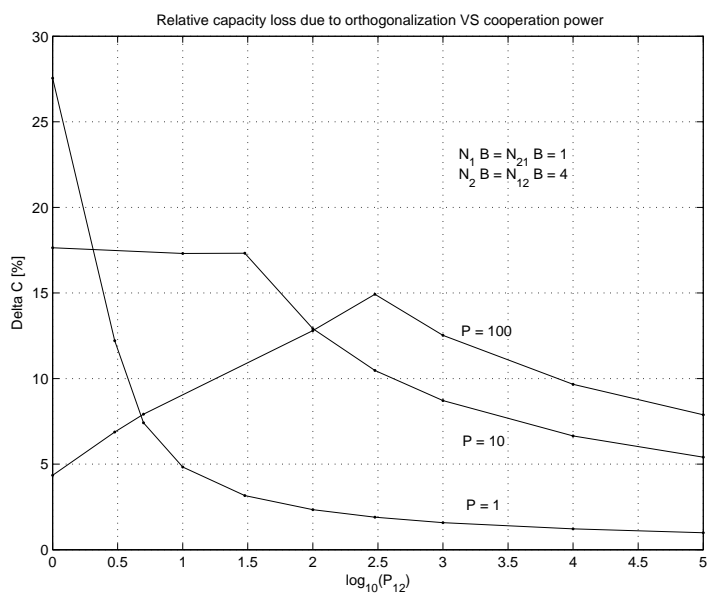

Fig. 2. Relative capacity loss versus $\log _{10}\left(P_{12}\right)$

\section{GAUSSIAN CBC WITH BIDIRECTIONAL AND ORTHOGONAL COOPERATION CHANNEL}

In this section we focus on the case of the orthogonal cooperation channel. In the previous section we have seen that decode-andforward (DAF) is optimum in the unidirectional case. In the bidirectional case the optimum decoding scheme is not known and only lower and upper bounds for the channel capacity (discrete case) are available [4], [5], [8]. Note that it is not possible to apply the conventional DAF at both receivers. Otherwise one cooperation direction would not be used since one of the receiver would have to decode reliably by himself. One of the purposes of this section is to use the natural extension of the lower and upper bounds of [4] for the discrete case, and apply them in the Gaussian case. In [4] the authors used estimate-and-forward (EAF) at both receivers to derive an achievable rate [5]. One of the disadvantages of this solution is that it is not capacity-achieving in the unidirectional case i.e. when $P_{21} \rightarrow 0$. Said otherwise using EAF at both receivers can be not efficient in low cooperation regimes. This is why we propose comparing this coding scheme with a coding scheme combining EAF and DAF. We note that [4] also proposed an hybrid coding scheme of this kind. The authors exploited the approach of multi-step decoding introduced by [8] for the discrete case. It turns out that the corresponding theorems of [4] are not easy to apply in the Gaussian case. Equally a similar scheme has been used recently to derive an achievable rate region for the discrete $\mathrm{CBC}$ with independent and common messages [5]. Here we specialize this approach by requiring one receiver to use DAF a fraction of the time and EAF for the rest of the time. The other receiver makes use of the "complementary" coding scheme for the corresponding periods of time.

\subsection{EAF at Rx1 plus EAF at $R x 2$}

As in section 3 we assume that the triplet $\underline{\alpha}$ is not fixed and therefore can be optimally chosen. From Theorem 4 of [4] one can show (the proof is omitted here because of the lack of space) that using EAF at both receivers in the Gaussian case with orthogonal cooperation channel translates into the following achievable rate: $R^{e a f}=$ $\max _{\left(\alpha_{0}, \alpha_{12}, \alpha_{21}\right)} \min \left\{R_{1}^{\text {eaf }}\left(\alpha_{0}, \alpha_{12}\right), R_{2}^{\text {eaf }}\left(\alpha_{0}, \alpha_{21}\right)\right\}$ with $\forall(i, j) \in$
$\{1,2\}^{2}: R_{i}^{\text {eaf }}\left(\alpha_{0}, \alpha_{i j}\right)=\alpha_{0} B \log _{2}\left(1+\frac{P}{\alpha_{0} N_{j} B}+\frac{P}{\alpha_{0}\left(N_{i}+\hat{N}_{i}\right) B}\right)$
with $\alpha_{0} \hat{N}_{i} B=\left[\alpha_{0} N_{i} B+\frac{P \alpha_{0} N_{j} B}{P+\alpha_{0} N_{j} B}\right]\left[\left(1+\frac{\rho_{i j}}{\alpha_{i j}}\right)^{\alpha_{i j} / \alpha_{0}}-1\right]^{-1}$ The noise power spectral densities $\hat{N}_{i}$ are defined with respect to the estimate-and-forward scheme: Receiver "i" sends an estimation $\hat{Y}_{i}=Y_{i}+\hat{Z}_{i}$ of its received signal to the other receiver. In the maximization above there are only two free parameters since the total bandwidth constraint imposes $\alpha_{0}+\alpha_{12}+\alpha_{21}=1$. For instance one can optimize the rate over $\left(\alpha_{0}, \alpha_{12}\right)$ by imposing $\alpha_{0} \leq 1, \alpha_{0}+$ $\alpha_{12} \leq 1, \alpha_{21}=1-\alpha_{0}-\alpha_{12}$. This coding scheme is capacityachieving in the two extreme cooperation regimes. When $\rho_{i j}=$ $P_{i j} /\left(N_{j} B\right) \rightarrow \infty$ one gets the Gaussian SIMO channel capacity. When $\rho_{i j}=0$ one gets $R^{\text {eaf,* }}=C_{\text {orth }}=\min \left\{C\left(\rho_{1}\right), C\left(\rho_{2}\right)\right\}$. Note that for "small" values of the transmit power, the rate has to be a concave function of $P$. In all the (typical) simulation scenarios presented in this paper, we did not use this "convexification" [9] and for sake of clarity we did not indicate the underlying optimization.

\subsection{EAF/DAF at $R x 1$ plus DAF/EAF at $R \times 2$}

First let us describe the decoding "protocol". Without loss of generality assume receiver 1 implements DAF and user 2 implements EAF. The transmitter sends a data block, none of the users can decode the message reliably from this block alone. User 2 sends an estimate of its received signal (through a data block) to user 1. User 1 is then able to decode at a rate $I\left(X ; Y_{1}, \hat{Y}_{2}\right)$. After decoding user 1 sends a message allowing receiver 2 to resolve its ambiguity about the transmitted message (list coding/decoding) and then able to decode at the rate $I\left(X ; Y_{2}\right)+I\left(X_{12} ; Y_{12}\right)$. The achievable rate is the minimum of the two aforementioned rates. At the end of the cooperation, receiver 1 and 2 used respectively 2 and 3 blocks for decoding.

As mentioned in the introducing part of this section, we need to introduce a time sharing parameter to decide how long receiver 1 uses DAF or EAF. Let $\tau=1-\bar{\tau}$ denote the time fraction in which receiver 1 uses EAF. The proposed hybrid scheme achieves the following rate:

$R^{h}=\max _{\tau, \underline{\alpha}} \min \left(\tau R_{1}^{e a f}(\underline{\alpha})+\bar{\tau} R_{1}^{d a f}(\underline{\alpha}), \tau R_{2}^{d a f}(\underline{\alpha})+\bar{\tau} R_{2}^{e a f}(\underline{\alpha})\right)$ with $R_{i}^{d a f}\left(\alpha_{0}, \alpha_{i j}\right)=\min \left\{\alpha_{0} C\left(\frac{\rho_{i}}{\alpha_{0}}\right), \alpha_{0} C\left(\frac{\rho_{j}}{\alpha_{0}}\right)+\alpha_{i j} C\left(\frac{\rho_{i j}}{\alpha_{i j}}\right)\right\}$.

In practice the best $\tau$, say $\tau^{*}$, is 0 or 1 in most cases. The special cases where $\tau^{*} \neq 0,1$ can be easily found. Let assume $\underline{\alpha}$ to be fixed and omit it from the notations for sake of simplicity. The maximization has the following simple form 
$R^{h}=\max _{\tau} \min \left\{\left(e_{1}-d_{1}\right) \tau+d_{1},\left(d_{2}-e_{2}\right) \tau+e_{2}\right\}$ where $e_{i}, d_{i}$ stands for the rates associated with EAF and DAF respectively. It appears that $\tau^{*}$ is different from 0 or 1 when $\left\{d_{1}<e_{1}, d_{2}<e_{2}, d_{1}<\right.$ $\left.\left.e_{2}\right)\right\}$ or $\left.\left\{d_{1}>e_{1}, d_{2}>e_{2}, d_{1}>e_{2}\right)\right\}$. These special regimes will occur in extreme cooperation regimes in which the hybrid scheme is not the most efficient coding/decoding scheme. However, in intermediate cooperation regimes we are interested in, the optimum fraction of time will be always zero or one. One can then simplify, without loss of performance in the considered regimes, the hybrid scheme into: $\tilde{R}^{h}=\max \left\{\min \left(R_{1}^{\text {eaf }}, R_{2}^{\text {daf }}\right), \min \left(R_{1}^{\text {daf }}, R_{2}^{\text {eaf }}\right)\right\}$. For example, when $e_{1}<d_{1}, e_{2}<d_{2}$ the hybrid scheme achieves $\max \left\{e_{1}, e_{2}\right\}$ instead of $\min \left\{e_{1}, e_{2}\right\}$ for the pure EAF-based decoding scheme.

\subsection{Upper bound for the Gaussian CBC rate}

Theorem 7 of [4] for the discrete CBC with a conference link can be extended to the Gaussian CBC with an orthogonal cooperation channel. The corresponding upper bounds can be shown to be:

$$
\begin{aligned}
R_{u p}= & \max _{\underline{\alpha}}\left\{\alpha_{0} C\left(\frac{\rho_{1}}{\alpha_{0}}\right)+\alpha_{21} C\left(\frac{\rho_{21}}{\alpha_{21}}\right),\right. \\
& \left.\alpha_{0} C\left(\frac{\rho_{2}}{\alpha_{0}}\right)+\alpha_{12} C\left(\frac{\rho_{12}}{\alpha_{12}}\right), \alpha_{0} C\left(\frac{\rho_{e q}}{\alpha_{0}}\right)\right\}
\end{aligned}
$$

where $\rho_{e q}=P\left(N_{1}+N_{2}\right) / N_{1} N_{2}$.

\subsection{Numerical results}

Here we want to compare the two coding/decoding schemes considered previously. Simulation setup: $N_{1} B=N_{21} B=1, N_{2} B=$ $N_{12} B=4, P=10, P_{12}=P_{21}$ with $P_{12} / P \in[-10 \mathrm{~dB}, 80 \mathrm{~dB}]$. Figure 3 represents the considered upper and lower bounds (normalized wrt $B$ ) for the rate as a function of $\log _{10}\left(P_{12}\right)$. The hybrid scheme performs better than the simple association of two EAFs. Of course, when no cooperation is possible, the two schemes are equivalent. For very high cooperation powers the hybrid scheme would be less efficient than the EAF-based scheme. To sum up, for typical values of $P_{12} / P<30-40 \mathrm{~dB}$, as those encountered in practice, the simplified hybrid solution is clearly the most efficient coding/decoding scheme. For this typical working range we also note that the relative performance loss of the simplified hybrid scheme with respect to the upper bound is reasonably small $(<6 \%)$.

\section{CONCLUSION}

From the provided coding theorem for the unidirectional CBC we have seen that orthogonalizing the cooperation channel leads to a relatively small performance loss $(<10 \%)$ for reasonably high power levels $\frac{P_{12}}{P}>20 \mathrm{~dB}$. On the other hand the non-orthogonal Gaussian $\mathrm{CBC}$ reaches its maximum performance for finite cooperation powers and is therefore much more efficient than its orthogonal counterpart in low cooperation regimes. For the Gaussian CBC with a bidirectional and orthogonal channel we have seen that the hybrid coding scheme using both decode-and-forward and estimate-andforward schemes performs very well for the cooperation regimes of practical interest. Although the optimum coding/decoding scheme is not known for the Gaussian BC with a single common message and a bidirectional (and othogonal) cooperation channel, the performance loss with respect to the upper bound under consideration is shown to be less than $6 \%$ for $\frac{P_{12}}{P}=\frac{P_{21}}{P}<40 \mathrm{~dB}$. As a conclusion, it would be interesting to extend these results in the fading and MIMO cases.

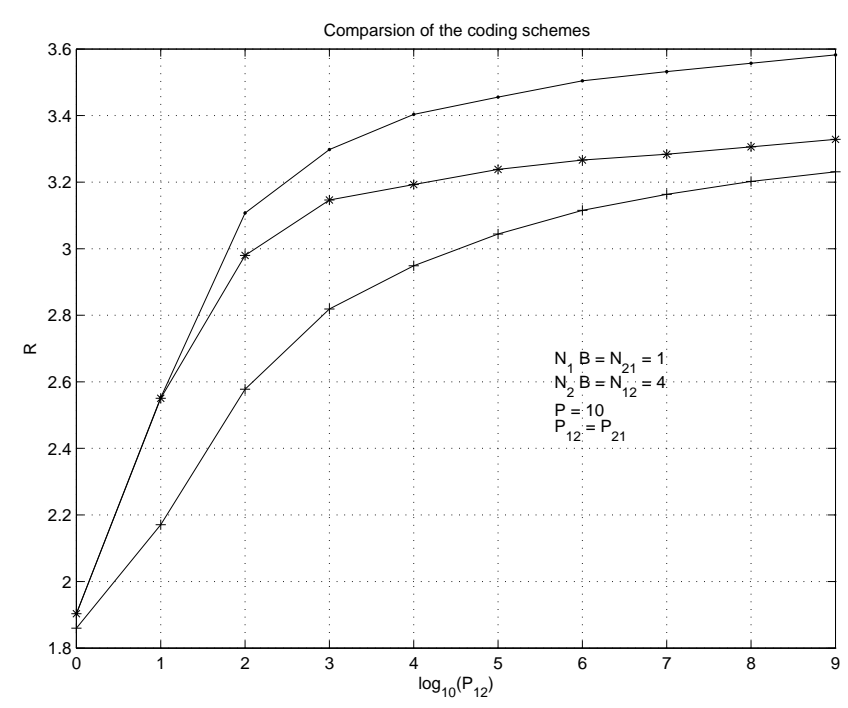

Fig. 3. (Up. bound, $\{$ eaf $1+$ eaf 2$\}$, hyb. scheme) vs $\log _{10}\left(P_{12}\right)$

\section{ACKNOWLEDGEMENTS}

The authors warmly thank Prof. Sergio Servetto for his valuable comments and insights on this work. We also would like to acknowledge Prof. Pierre Duhamel and Prof. Rick Johnson for their roles in facilitating the international collaboration that brought about this work.

\section{REFERENCES}

[1] F. Willems, "The discrete memoryless multiple access channel with partially cooperating encoders", IEEE Trans. Inform. Theory, IT-29(5): 441-445, 1983.

[2] A. Sendonaris, E. Erkip, and B. Aazhang, "User cooperation diversity-Part I: System description", IEEE Trans. on Comm., COM-51(11): 1927-1938, 2003.

[3] J. N. Laneman, D. N. C. Tse, and G. W. Wornell, "Cooperative diversity in wireless networks: Efficient protocols and outage behavior", IEEE Trans. Inform. Theory, IT-50(12): 3063-3080.

[4] R. Dabora and S. D. Servetto, "Broadcast channels with cooperating decoders", sub. to IEEE Trans. Inform. Theory in May 2005.

[5] Y. Liang and V. V. Veeravalli, "Cooperative relay broadcast channels", Proc. IEEE WirelessCom, Symposium on Information Theory, Maui, Hawai, June 2005.

[6] Y. Liang and V. V. Veeravalli, "Gaussian orthogonal relay channel: Optimal resource allocation and capacity", IEEE Trans. Inform. Theory, IT-51(9):3284-3289, Sep. 2005.

[7] T. M. Cover and A. A. El Gamal, "Capacity theorems for the relay channel", IEEE Trans. Inform. Theory, IT-25(5): 572-584.

[8] S. C. Draper, B. J. Frey, and F. R. Kschischang, "Interactive decoding of a broadcast message", Proc. IEEE Allerton Conf. Commun., Contr., Computing, Oct. 2003.

[9] A. El Gamal, M. Mohseni, and S. Zahedi, "On reliable communication over additive white Gaussian noise relay channels", submitted to IEEE Trans. Inform. Theory in Sep. 2004. 